\title{
Opioid-Free Anesthesia for Laparoscopic Hysterectomy: Is it Appropriate?
}

\author{
${ }^{1}$ Department of Anesthesiology \& ICU, Benha University, Egypt \\ ${ }^{2}$ Department of Anesthesiology \& ICU, Ministry of Health, Egypt \\ ${ }^{3}$ Department of Obstetrics \& Gynecology, Benha University, Egypt \\ ${ }^{4}$ Department of Obstetrics \& Gynecology, Tanta University, Egypt
}

Ahmed M Salem ${ }^{1 *}$, Mohamed ML Hafez ${ }^{2}$, Amr Sharaf Eldin ${ }^{3}$ and Ahmed M Hagras

Submission: July 01, 2019; Published: July 18, 2019

*Corresponding author: Ahmed M Salem, Department of Anesthesiology \& ICU, Faculty of Medicine, Benha University, Egypt

\begin{abstract}
Objective: To evaluate intraoperative (IO) and postoperative (PO) outcome of women assigned to laparoscopic hysterectomy under opioidfree anesthesia (OFA) in comparison to opioid-based anesthesia (OBA).

Patient and Methods: 72 women were randomly divided into OBA and OFA groups according to the provided IO analgesic regimen. OBA patients received Fentanyl (FEN) as loading and Remifentanyl (REM) infusion as maintenance analgesia. OFA patients received preoperative parecoxib sodium for preparation, dexmedetomidine (DEX) and lidocaine (LID) as loading and maintenance analgesia. Study Outcomes included the frequency of patients developed IO deviated mean arterial pressure (MAP) measures by $>20 \%$ of baseline measures, duration of surgery and time till fulfilling criteria for PACU discharge, duration of PO analgesia, time till 1st ambulation, PO complications and hospital stay.

Results: Demographic data and surgical characteristics were comparable in all groups. The D group showed delay in onset for first call for analgesia $(900 \pm 60.9 \mathrm{~min})$ while $\mathrm{M}$ and $\mathrm{K}$ groups results were $(600 \pm 33.4 \mathrm{~min})$ and $(350 \pm 17.4 \mathrm{~min})$ respectively, all of the group's results were statistically significant than the control group result $(260 \pm 14.3 \mathrm{~min})$.

Results: MAP measures during and 30-min after abdominal insufflations were significantly higher in patients of OFA than patients of OBA. Among 216 MAP readings, increased MAP measures by $>20 \%$ of baseline measure was recorded in $10(4.6 \%)$ versus $3(1.4 \%)$ readings in OFA and OBA groups, respectively with a non-significant difference $(\mathrm{p}=0.091)$ between both groups. Duration till 1 st ambulation was significantly shorter with OFA compared to OBA, while duration till 1st request of rescue analgesia was significantly longer with OFA, while the frequency of patients requested more rescue analgesia was significantly higher with OBA than OFA. PONV was reported in 53 patients and 17 patients required anti-emetic therapy with significantly higher incidence with OBA compared to OFA group.
\end{abstract}

Conclusion: The applied protocol for OFA provided satisfactory IO analgesia and control of surgery-induced pressor reflexes. Also, it allowed reduction of PO analgesic consumption with early ambulation and reduced PONV that were reflected as shorter PO hospital stay.

Keywords: Opioid-free anesthesia; Parecoxib; Dexmedetomidine; Lidocaine; Remifentanyl; Laparoscopic hysterectomy

\section{Introduction}

Hysterectomy is the most common major gynecological procedure in women [1], but hysterectomy for enlarged uteri is considered a challenge for gynecologic surgeons, due to the limit of exposure to surgical spaces [2]. Minimally invasive approaches as total laparoscopic hysterectomy should be used wherever possible [1] as it is the standard of care in majority of women diagnosed with endometrial cancer [3] and is feasible for enlarged uteri [2]. Postoperative (PO) pain remains one of the most common challenges following inpatient and outpatient surgeries [4]. Opioids are the most potent drugs used to control severe pain [5], however, surgical care episodes place opioid-naive patients at risk for transitioning to new persistent PO opioid use [6].
Opioids are frequently associated with adverse events such as dizziness, drowsiness [7], high incidence of PO nausea and vomiting, which varies from $20 \%-60 \%$ [8] or constipation which disturbs PO recovery and extends the duration of hospital stay [7].

With increased awareness of both short- and longterm problems associated with liberal perioperative opioid administration, the need for routinely and clinically feasible alternatives is greater than ever [9]. Implementation of multimodal analgesic regimen achieved equivalent and effective pain control aiming to reduce the reliance on opioid-based medications [10]. The application of enhanced recovery pathways promoted opioid-free and multimodal analgesia [11] and allowed 
decreased perioperative opioid use [6]. Opioid-free anesthesia (OFA) has gained in popularity to enhance early recovery and to spare opioid use for the PO period [12]. OFA was found to be safe, provided comfort during the immediate PO period equal to after conventional anesthesia and may provide reduced pain during the first 24 PO hours [13].

Dexmedetomidine (DEX) is $\alpha 2$-adrenergic sedative-hypnotic medication [14] with analgesic, sedative and sympatholytic properties and a lack of respiratory depression [15], so it is used as an adjunct to general anesthesia [14]. Also, DEX can effectively reduce the incidence of $\mathrm{PO}$ cognitive impairment with high safety for circulatory function [16].

\section{Hypothesis}

Opioid-free anesthesia improves postoperative outcome of women underwent laparoscopic hysterectomy and hastens recovery and home-discharge without compromising operative outcome.

\section{Objective}

Evaluation of intraoperative (IO) and PO outcome of women assigned to laparoscopic hysterectomy under OFA compared to opioid-based anesthesia (OBA).

\section{Setting}

Departments of Anesthesia and Gynecology, Benha \& Tanta Universities, Ministry of Health, Tanta, Egypt.

\section{Design}

Prospective clinical trial.

\section{Patients and Methods}

The study protocol was approved by the Local Ethical Committees and patient and her husband signed written fully informed consents according to the declaration of Helsinki. All women assigned for hysterectomy were eligible for evaluation, patients with ASA grade $>$ II, body mass index (BMI) $>35 \mathrm{~kg} / \mathrm{m}^{2}$, cardiac or chest diseases, previous open abdominal surgery, history of treatment or surgery for hiatus hernia, hypersensitivity to the drugs to be used, requiring total hysterectomy or pelvic excentration or refusing laparoscopic surgery were excluded from the study. All eligible women were clinically evaluated for demographic and baseline clinical data collection, underwent laboratory and radiological workup for assurance of inclusion and exclusion criteria.

\section{Randomization and grouping}

Patients were randomly allocated into two groups; control group included women assigned to receive opioid-based anesthesia (OBA group), while study group included women assigned to receive opioids-free anesthesia (OFA group). For randomization, cards carrying group label were prepared by an assistant blinded about the significance of the label and cards were put in envelops free of marks and closed. On arrival to preanesthetic room, patient was asked to choose a card that was opened by anesthetist in charge.

\section{Preparation of Study drugs}

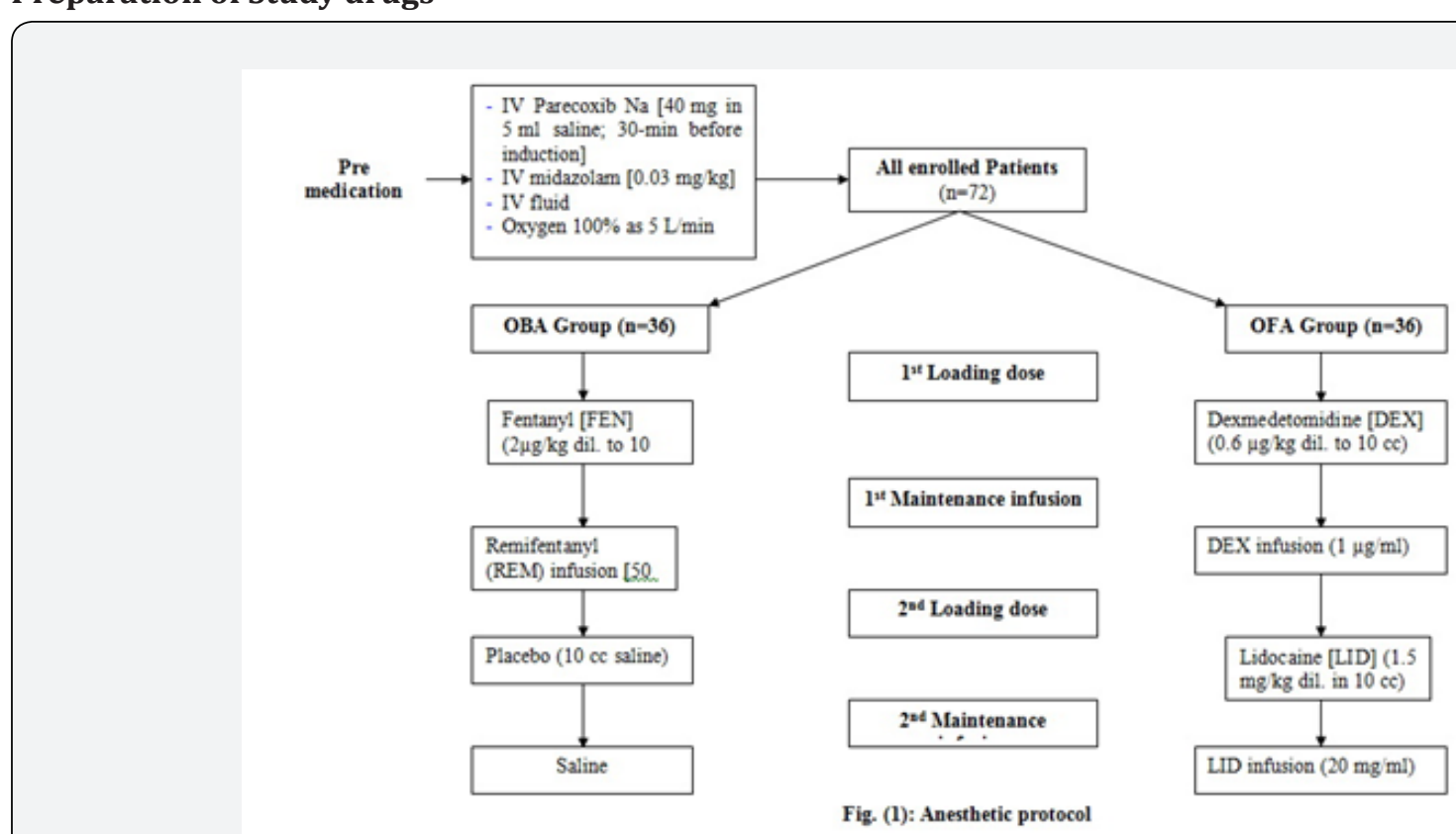

Figure 1: Anesthetic protocol.

Study drugs were freshly prepared by hospital clinical pharmacist blinded about the significance of labels. Drugs were prepared according to the protocol that previously documented by Bakan et al. [17]. For each group, two 10-cc syringes were prepared to provide the loading dose (L-1 \& L-2) and two infusion fluid bottles for maintenance of anesthesia (M-1 \& M-2) 
a) Opioid-based anesthesia (OBA) group: Fentanyl (FEN) loading dose was prepared as $2 \mu \mathrm{g} / \mathrm{kg}$, diluted to a total volume of $10 \mathrm{cc}$ in a syringe labeled as CL-1 and CL-2 syringe was filled by 10 -cc saline as placebo. Remifentanyl (REM) infusion was prepared as $50 \mu \mathrm{g} / \mathrm{ml}$, was labeled as $\mathrm{CM}-1$ and a bottle of saline free of additives was prepared as CM-2 infusion.

b) Opioid-free anesthesia (OFA) group: Dexmedetomidine (DEX) loading dose was prepared as $0.6 \mu \mathrm{g} / \mathrm{kg}$, diluted to a total volume of $10 \mathrm{cc}$ in a syringe labeled as SL-1 and Lidocaine (LID) loading dose was prepared as $1.5 \mathrm{mg} / \mathrm{kg}$ in a syringe labeled SL-2. DEX infusion was prepared as $1 \mu \mathrm{g} / \mathrm{ml}$, was labeled as SM-1 and LID infusion was prepared as $20 \mathrm{mg} / \mathrm{ml}$ was labeled as SM-2 (Figure 1).

\section{Anesthetic protocol}

a) At pre-anesthetic room, baseline heart rate (HR) and mean arterial blood pressure (MAP) were determined noninvasively. Parecoxib sodium $40 \mathrm{mg}$ diluted with $5 \mathrm{ml}$ of saline was injected intravenously (IV) 30-min before induction of anesthesia. Patients were premedicated with IV midazolam $(0.03 \mathrm{mg} / \mathrm{kg})$, received IV fluid and were maintained welloxygenated using oxygen $100 \%$ as $5 \mathrm{~L} / \mathrm{min}$ flow rate.

b) On arrival to the theater, patients of both groups received the prepared drugs according the chart shown in Figure 1 as follows:

c) 1st loading doses (CL-1 and SL-1 syringes containing Fen \& DEX loading dose, respectively) were injected in 10 $\min$.

d) CM-1 and SM-1 infusions of REM \& DEX, respectively, were started at rate of $0.3 \mathrm{ml} / \mathrm{kg} / \mathrm{h}$.

e) 2nd loading doses (CL-2 and SL-2 syringes containing plain saline \& LID, respectively) were infused simultaneously with induction dose of propofol $(1.5 \mathrm{mg} / \mathrm{kg})$

f) Immediately, CM-2 and SM-2 infusions of plain saline and LID, respectively were started simultaneously with propofol infusion at rate of $10 \mathrm{mgl} / \mathrm{kg} / \mathrm{h}$.

g) IV rocuronium $(0.6 \mathrm{mg} / \mathrm{kg})$ was injected to facilitate tracheal intubation.

h) After intubation of the trachea, the lungs were ventilated with $100 \% 02$ in air using a semi-closed circle system. During surgery, ventilation was controlled with a tidal volume of $6-8 \mathrm{ml} / \mathrm{kg}$, and the ventilatory rate was adjusted to maintain an end-tidal carbon dioxide (paCO2) of 32-35mmHg. Patients were continuously non-invasively monitored for MAP and HR.

i) Infusions $\mathrm{M}-1$ and $\mathrm{M}-2$ were maintained at the initial rate, but propofol infusion was adjusted to $3-12 \mathrm{mg} / \mathrm{kg} / \mathrm{hr}$ to maintain HR and MAP within $\pm 20 \%$ of baseline MAP till skin closure. j) After skin closure, any residual neuromuscular blockade was reversed by neostigmine $0.05 \mathrm{mg} / \mathrm{kg}$ and atropine $0.02 \mathrm{mg} / \mathrm{kg}$ and tracheal extubation was performed when patients achieved a regular spontaneous breathing and patients were transferred to the post-anesthesia care unit (PACU).

k) At PACU, oxygen saturation was monitored using pulse oximetry and oxygen $(6 \mathrm{~L} / \mathrm{min})$ was administrated via a facemask in the PACU if indicated. PACU discharge was dependent on Aldrete recovery score that ranges from 0 (comatose patients) to 10 (complete recovery), patients were discharged at score of $\geq 8$ [18].

\section{Postoperative care}

Postoperative pain severity was assessed using an 11-point numeric rating scale (NRS) with numbers from 0 to 10 where 0 indicates no pain and 10 indicates worst pain imaginable [19]. PO pain was assessed at time of PACU discharge before the 2nd dose of parecoxib and 4-hourly for 24-hr. Duration of PO analgesia was defined as time till 1st request of rescue analgesia that was supplied as IV parecoxib (20mg diluted in 5cc saline). Frequency of requests of rescue analgesia was also determined.

\section{Study Outcomes}

\section{Primary outcome}

a) The frequency of patients developed IO deviated MAP measures by $>20 \%$ of baseline measures and necessitated interference.

\section{Secondary outcome}

a) IO mean HR and MAP determined before and after intubation and at time of pneumoperitoneum, and five minutely till uterus extraction

b) Duration of surgery, anesthesia and time till fulfilling criteria for PACU discharge

c) Frequency of requests of rescue analgesia, time till 1st ambulation, PO complications and PO hospital stay.

\section{Results}

One hundred and seven patients were eligible for evaluation, 35 women were excluded, 72 women were equally divided into two groups (Figure 2). There was non-significant ( $p>0.05$ ) difference between both groups as regards enrolment data determined at time of enrolment as shown in Table 1.

Heart rate and MAP measures that were recorded preoperatively, before induction of anesthesia and after intubation showed non-significant difference between patients of both groups. HR measures were increased during abdominal insufflation in patients of both groups but were non-significantly higher in patients of OFA group than those of OBA group. During surgery and at time of extubation HR measures were non- 


\section{Journal of Anesthesia \& Intensive Care Medicine}

significantly higher in patients of OFA group. MAP measures during and 30-min after abdominal insufflation were significantly higher ( $p=0.041 \& 0.0002$, respectively) in patients of OFA than patients of OBA, while at 45-min after insufflation and at time of extubation, MAP measures were non-significantly higher in OFA group compared to OBA group (Table 2).

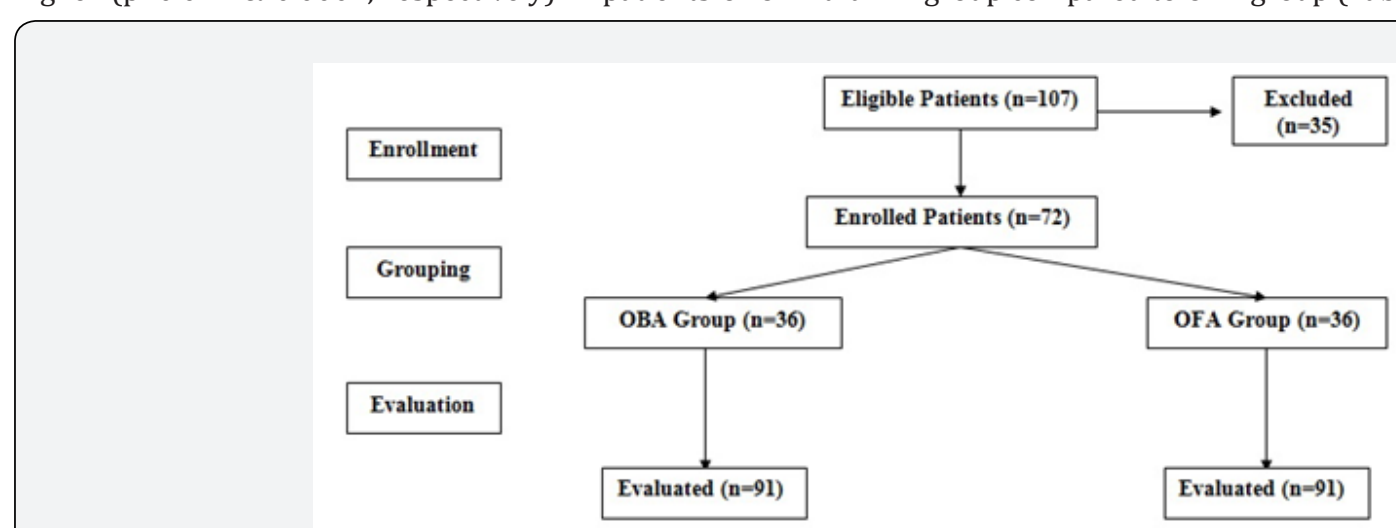

Figure 2: Flow chart of the study.

Table 1: Baseline data of enrolled women.

\begin{tabular}{|c|c|c|c|c|}
\hline \multicolumn{2}{|c|}{ Data } & OBA $(n=36)$ & OFA $(n=36)$ & $P$ value \\
\hline \multicolumn{2}{|c|}{ Age (years) } & $52.4 \pm 8.1$ & $50.4 \pm 6.6$ & 0.371 \\
\hline \multirow{3}{*}{ BMI data } & Weight (kg) & $81.5 \pm 7.9$ & $82.5 \pm 8.8$ & 0.595 \\
\hline & Height $(\mathrm{cm})$ & $169.1 \pm 4.2$ & $169.5 \pm 3.2$ & 0.661 \\
\hline & BMI $\left(\mathrm{kg} / \mathrm{m}^{2}\right)$ & $28.5 \pm 2.9$ & $28.7 \pm 3.1$ & 0.463 \\
\hline \multirow{2}{*}{ ASA grade } & ASA-I & $28(77.8 \%)$ & $26(72.2 \%)$ & 0.552 \\
\hline & ASA-II & $8(22.2 \%)$ & $10(27.8)$ & \\
\hline \multirow{2}{*}{ Indication for surgery } & Fibroid & $21(58.3 \%)$ & $23(63.9 \%)$ & 0.629 \\
\hline & DUB & $15(41.7 \%)$ & $13(36.1 \%)$ & \\
\hline \multicolumn{2}{|c|}{ Fasting blood glucose (mg/dl) } & $82.7 \pm 8.4$ & $84.1 \pm 6$ & 0.078 \\
\hline
\end{tabular}

Data are presented as mean \pm SD, numbers, percentages; BMI: Body mass index; DUB: Dysfunctional uterine bleeding; $P$ indicates the significance of difference between both groups; $p>0.05$ : indicates non-significant difference.

Table 2: Preoperative and intraoperative HR and MAP measures of patients of both groups.

\begin{tabular}{|c|c|c|c|c|}
\hline Variable & Time & OBA $(n=36)$ & OFA $(n=36)$ & P Value \\
\hline \multirow{8}{*}{ Heart Rate (beats/min) } & Preoperative & $82.8 \pm 4$ & $80.7 \pm 6.3$ & 0.103 \\
\hline & At time of induction & $76.2 \pm 4.7$ & $74.5 \pm 5.3$ & 0.159 \\
\hline & At time of intubation & $84.5 \pm 3.9$ & $86.4 \pm 4.6$ & 0.061 \\
\hline & Before abdominal insufflation & $70 \pm 6.2$ & $72.3 \pm 6.5$ & 0.128 \\
\hline & At time of abdominal insufflation & $73 \pm 5.4$ & $75.2 \pm 7.1$ & 0.151 \\
\hline & 30-min after abdominal insufflation & $72 \pm 4.6$ & $73.3 \pm 6.1$ & 0.311 \\
\hline & 45-min after abdominal insufflation & $74.4 \pm 7.3$ & $76.4 \pm 7.5$ & 0.243 \\
\hline & At time of extubation & $76.4 \pm 7.5$ & $79.5 \pm 7$ & 0.077 \\
\hline \multirow{8}{*}{ MAP (mmHg) } & Preoperative & $88.9 \pm 3$ & $89.5 \pm 3.1$ & 0.369 \\
\hline & At time of induction & $92.3 \pm 2.8$ & $93.1 \pm 3$ & 0.237 \\
\hline & At time of intubation & $92.6 \pm 2.9$ & $93.5 \pm 2.7$ & 0.207 \\
\hline & Before abdominal insufflations & $94.8 \pm 2.7$ & $96 \pm 5.5$ & 0.225 \\
\hline & At time of abdominal insufflations & $95 \pm 2.4$ & $96.6 \pm 4.4$ & 0.041 \\
\hline & 30-min after abdominal insufflations & $90.5 \pm 6.6$ & $95.2 \pm 2.8$ & 0.0002 \\
\hline & 45-min after abdominal insufflations & $93.2 \pm 2.3$ & $92.8 \pm 2.9$ & 0.467 \\
\hline & At time of extubation & $92.6 \pm 2.7$ & $93.5 \pm 2.7$ & 0.141 \\
\hline
\end{tabular}

Data are presented as mean $\pm S D ; P$ indicates the significance of difference between both groups; $p>0.05$ : indicates non-significant difference; $p<0.05$ : indicates significant difference. 


\section{Journal of Anesthesia \& Intensive Care Medicine}

Considering the primary outcome, all patients of OFA group showed increased MAP measures recorded throughout operative time in relation to baseline MAP. However, MAP estimated just before abdominal insufflations was increased by $>20 \%$ of baseline measure in 3 patients $(8.3 \%)$, in 5 patients $(13.9 \%)$ at time of abdominal insufflations and in one patient $(2.8 \%)$ at 30 and 45 min after abdominal insufflations. Thus, increased MAP measures by $>20 \%$ of baseline measure was recorded in 10 readings of 216 readings for a rate of $4.6 \%$. Among, patients of OBA group, MAP was increased by $>20 \%$ of baseline measure in three readings only (1.4\%), while was decreased than baseline measures in 12 readings $(5.6 \%)$. Comparison of percentage of MAP change in relation to baseline measures in both groups, the difference was significant $(\mathrm{p}=0.008)$ only at 30 -min after abdominal insufflations secondary to development of decreased MAP in patients of OBA group. All other calculated percentages of MAP changes showed non-significant difference between patients of both groups (Table 3 , Figure 3).

Table 3: Percentage of change of intraoperative MAP measures of patients of both groups in relation to their preoperative measures.

\begin{tabular}{|c|c|c|c|}
\hline Time & OBA $(n=36)$ & OFA $(n=36)$ & P Value \\
\hline At time of induction & $3.9 \pm 1.4$ & $4 \pm 1.6$ & 0.619 \\
\hline At time of intubation & $4.2 \pm 1.6$ & $4.4 \pm 1.4$ & 0.575 \\
\hline Before abdominal insufflations & $6.8 \pm 5.3$ & $7.4 \pm 6.9$ & 0.678 \\
\hline At time of abdominal insufflations & $6.9 \pm 4.3$ & $8.1 \pm 6.3$ & 0.352 \\
\hline 30-min after abdominal insufflations & $2 \pm 8.5$ & $6.4 \pm 4.7$ & 0.008 \\
\hline 45-min after abdominal insufflations & $5 \pm 4.7$ & $3.7 \pm 3.1$ & 0.185 \\
\hline At time of extubation & $4.2 \pm 1.7$ & $4.5 \pm 1.6$ & 0.441 \\
\hline
\end{tabular}

Data are presented as mean $\pm S D ; P$ indicates the significance of difference between both groups; $p>0.05$ : indicates non-significant difference; $\mathrm{p}<0.05$ : indicates significant difference.

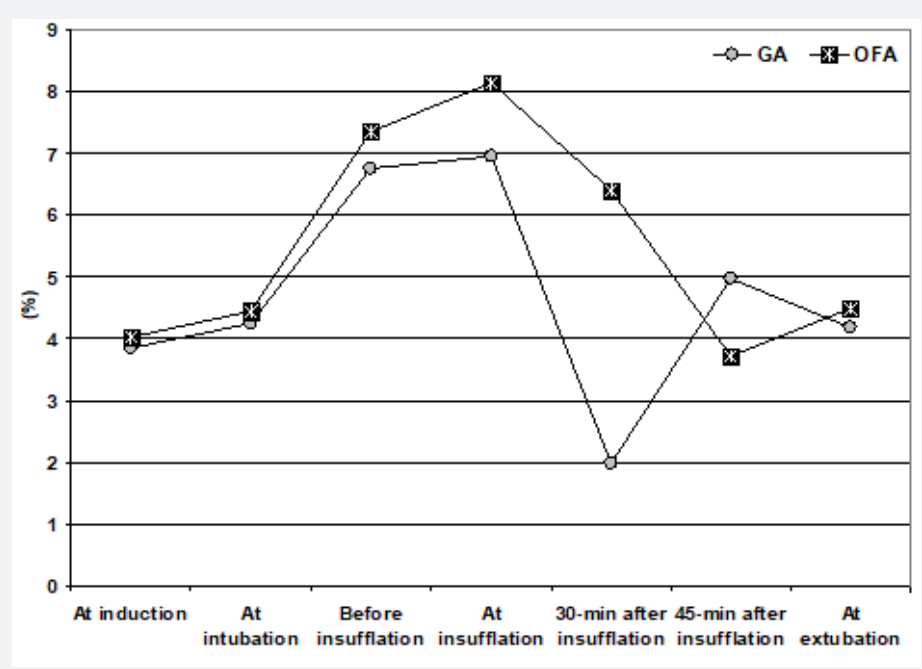

Figure 3: Mean percentage of change of MAP in relation to base line MAP.

Mean operative time and time for being ready to discharge to PACU were non-significantly shorter, while duration till 1st ambulation was significantly shorter with OFA compared to OBA. On contrary, duration till 1 st request of rescue analgesia was significantly longer with OFA, while the frequency of patients requested more rescue analgesia was significantly higher with OBA than OFA. Only 18 patients complained of PO shivering; 12 with OBA and 6 with OFA with non-significantly higher frequency with OBA. On the other hand, 53 patients complained of $\mathrm{PO}$ nausea or nausea and vomiting; 31 in OBA and 22 in OFA groups, and 17 patients required anti-emetic therapy; 13 in OBA and 4 in OFA groups with significantly higher incidence with OBA compared to OFA group (Table 4).

Table 4: Operative and postoperative data of patients of both groups.

\begin{tabular}{|c|c|c|c|}
\hline Items & OBA (n=36) & OFA (n=36) & P Value \\
\hline Operative time (min) & $234 \pm 44.4$ & $227 \pm 50$ & 0.548 \\
\hline Time till PACU discharge (min) & $27.9 \pm 6.5$ & $25.1 \pm 5.5$ & 0.054 \\
\hline Duration till 1st ambulation (min) & $95.3 \pm 11.7$ & $85.4 \pm 14.7$ & 0.002 \\
\hline Duration till 1st request of rescue analgesia (min) & $66.6 \pm 13.9$ & $76.9 \pm 17.8$ & 0.007 \\
\hline
\end{tabular}


Journal of Anesthesia \& Intensive Care Medicine

\begin{tabular}{|c|c|c|c|c|c|}
\hline \multirow{3}{*}{\multicolumn{2}{|c|}{ Number of requests of rescue analgesia }} & One & $12(33.3 \%)$ & $20(55.5 \%)$ & \multirow{3}{*}{0.023} \\
\hline & & Two & $16(44.4 \%)$ & $15(41.7 \%)$ & \\
\hline & & Three & $8(22.3 \%)$ & $1(2.8 \%)$ & \\
\hline \multirow{6}{*}{ PO complications } & \multirow{2}{*}{ Shivering } & No & $24(66.7 \%)$ & $30(83.3 \%)$ & \multirow{2}{*}{0.174} \\
\hline & & Yes & 12 (33.3\%) & $6(16.7 \%)$ & \\
\hline & \multirow{2}{*}{ PONV } & No & $5(13.9 \%)$ & $14(38.9 \%)$ & \multirow{2}{*}{0.033} \\
\hline & & Yes & $31(86.1 \%)$ & $22(61.1 \%)$ & \\
\hline & \multirow{2}{*}{ Antiemetic therapy } & No & 23 (63.9\%) & $32(88.9 \%)$ & \multirow{2}{*}{0.026} \\
\hline & & Yes & $13(36.1 \%)$ & $4(11.1 \%)$ & \\
\hline
\end{tabular}

\section{Discussion}

Opioid-free anesthesia (OFA) ameliorated but could not totally control the pressor response to intubation and abdominal insufflations as manifested by increased MAP measures recorded throughout operative time in relation to baseline MAP. On contrary, opioid-based anesthesia (OBA) allowed better control for these pressor responses as evidenced by the decreased percentage of increased MAP in relation to preoperative MAP on comparison to patients who received OFA. However, the difference between percentages of MAP change recorded in patients of both groups was non-significant except at 30-min after insufflations.

These findings indicated proper control of OFA on surgical stimuli that may initiate pressor response and illustrated the feasibility of OFA as the sole anesthetic for various surgical procedures. In line of this assumption, Lavand'homme \& Estebe [12] suggested that OFA stands as a new paradigm, which can deliver safe and stable anesthesia without intraoperative (IO) opioids to patients undergoing various surgical procedures. Also, Leas et al. [20] reported that opioid-free multimodal pain management is safe and effective option in patients undergoing shoulder arthroplasty with a very low risk of requiring rescue opioids and Soffin et al. [21] found OFA within an ERAS pathway for lumbar spinal decompression can minimize perioperative opioid exposure without adversely affecting pain control or recovery. Moreover, Mulier [22] documented that opioid-free general anesthesia is a viable option for gynecological and breast surgeries. Furthermore, Mulier \& Dillemans [23] reported that OFA was associated with fewer complications and lower healthcare resource utilization for patients undergoing bariatric surgery.

The reported significant difference between the percentage of MAP change at 30-min after abdominal insufflations could be attributed to decreased MAP secondary to the hypotensive effect of remifentanil and not to exaggerated MAP in patients of OFA group. In line with this explanation, Bakan et al. [17] reported significantly higher frequency patients requiring ephedrine to treat hypotension in patients received remifentanil-based anesthesia compared to those received OFA. Recently, Grape et al. [24] in meta-analysis of studies compared IO remifentanil versus DEX and found rates of hypotension; shivering and postoperative nausea and vomiting (PONV) were twice as frequent in patients who received OFA.
Among 216 MAP readings, increased MAP measures by $>20 \%$ of baseline measure was recorded in $10(4.6 \%)$ versus 3 $(1.4 \%)$ readings in OFA and OBA groups, respectively with a nonsignificant difference $(p=0.091)$ between both groups. These findings illustrated the safety of OFA for laparoscopic surgery and the feasibility of this type of surgery under OFA. In support of this outcome, Díaz-Crespo et al. [25] presented a case scheduled for laparoscopic bariatric surgery who while maintaining OFA, was converted to open surgery with correct control achieved of both hemodynamics and perioperative pain. Recently, Frauenknecht et al. [26] documented that there is strong evidence that OBA does not reduce PO pain, but is associated with more PONV, when compared with OFA.

The reported beneficial effects of OFA could be attributed to varied effects of each of the used drugs; namely parecoxib sodium, dexmedetomidine and lidocaine, to induce and maintain OFA. Such attribution was in line with Guo et al. [27] who suggested that COX-2 inhibitor parecoxib exerts its analgesic effect on surgical pain through the inhibition of spinal cord neuronal extracellular signal-regulated kinase activation and Takaku et al. [28] experimentally found pretreatment with a single dose of parecoxib reduced the inflammatory response with attenuation of serum and tissue levels of IL- $1 \alpha$, IL-1 $\beta$, IL-6, IL-10, \& TNF- $\alpha$.

Regarding, effects of DEX, Funai et al. [29] experimentally suggested that systemic $\alpha 2$-adrenoceptor stimulation by DEX may facilitate inhibitory synaptic responses in the superficial dorsal horn to produce analgesia mediated by activation of the pontospinal noradrenergic inhibitory system. Also, Yamakita et al. [30] experimentally, suggested that DEX has a peripheral mechanism of anti-inflammatory action through inhibition of p38 MAPK phosphorylation via TNF- $\alpha$ and this provides a molecular basis for its preventive action on peripheral sensitization following surgery. Clinically, Jebaraj et al. [31] documented that intraoperative DEX has equal analgesic efficacy to fentanyl and can be used as the sole analgesic agent in patients undergoing robotic urological surgery and Zhang et al. [32] found DEX infusion significantly decreased morphine consumption during the first 24-h PO with concomitant decreased plasma cortisol and IFN- $\gamma /$ IL-10 levels, but increased percentages of CD8+ \& CD4+/CD8+ cells, so Zhang et al. [32], attributed the effect of DEX infusion to its modulatory effect on stress reactions during the perioperative period. Recently, Grape et al. [24] in meta-analysis of studies 
compared IO remifentanil versus DEX and found pain scores at 2-hr PO were lower, time to analgesia request was longer and use of PO morphine were less with DEX.

Concerning lidocaine, Cui etal. [33] attributed antihyperalgesia effects of systemic lidocaine to inhibition of phosphorylation of $\mathrm{Ca} 2+$ /calmodulin-dependent protein kinase II and protein expression levels in somatosensory cortical neurons. Also, Kurabe et al. [34] experimentally, attributed the analgesic action of IV lidocaine in acute pain to its inhibition of glutamate release from presynaptic terminals in spinal substantia gelatinosa neurons with concomitant hyper-polarization of postsynaptic neurons by shifting the membrane potential leading to decreased excitability of spinal dorsal horn neurons. Clinically, Nakhli et al. [35] found intravenous lidocaine infusion permitted a reduction of volatile anesthesia and IO opioid consumption during renal surgery, so it could provide effective strategy especially in low- and middleincome countries. Also, Soto et al. [36] reported that perioperative lidocaine infusion may be a useful analgesic adjunct in enhanced recovery protocols due to its immuno-modulatory properties over surgical stress and so suggested its use in the context of multimodal analgesia.

\section{Conclusion}

The applied protocol for OFA provided satisfactory IO analgesia and control of surgery-induced pressor reflexes. Also, it allowed reduction of PO analgesic consumption with early ambulation and reduced PONV that were reflected as shorter PO hospital stay. However, wider-scale comparative studies are mandatory to establish these outcomes.

\section{References}

1. Obermair A, Armfield NR, Graves N, Gebski V, Hanna GB, et al. (2019) How to train practising gynaecologists in total laparoscopic hysterectomy: protocol for the stepped wedge IMAGINE trial. 9(5): e027155.

2. Cianci S, Gueli Alletti S, Rumolo V, Rosati A, Rossitto C, et al. (2019) Total laparoscopic hysterectomy for enlarged uteri: factors associated with the rate of conversion to open surgery. J Obstet Gynaecol 39(6): 805-810.

3. Chambers LM, Carr C, Freeman L, Jernigan AM, Michener CM (2019) Does Surgical Platform Impact Recurrence and Survival? A Study of Utilization of Multi Port, Single Port and Robotic-Assisted Laparoscopyin Endometrial Cancer Surgery. Am J Obstet Gynecol S00029378(19): 30654-4.

4. Mitra S, Carlyle D, Kodumudi G, Kodumudi V, Vadivelu N (2018) New Advances in Acute Postoperative Pain Management. Curr Pain Headache Rep 22(5): 35.

5. Lavand'homme P, Steyaert A (2017) Opioid-free anesthesia opioid side effects: Tolerance and hyperalgesia. Best Pract Res Clin Anaesthesiol 31(4): 487-498.

6. Wu CL, King AB, Geiger TM, Grant MC, Grocott MPW, et al. (2019) Fourth Perioperative Quality Initiative Workgroup: American Society for Enhanced Recovery and Perioperative Quality Initiative Joint Consensus Statement on Perioperative Opioid Minimization in Opioid-Naive Patients. Anesth Analg

7. Omar E, Wallon G, Bauer C, Axiotis G, Bouix C (2019) Evaluation of intravenous lidocaine in head and neck cancer surgery: study protocol for a randomized controlled trial. Trials 20(1): 220 .
8. Cho E, Kim DH, Shin S, Kim SH, Oh YJ, et al. (2018) Efficacy of Palonosetron-Dexamethasone Combination Versus Palonosetron Alone for Preventing Nausea and Vomiting Related to Opioid-Based Analgesia: A Prospective, Randomized, Double-blind Trial. Int J Med Sci 15(10): 961-968.

9. Mauermann E, Ruppen W, Bandschapp 0 (2017) Different protocols used today to achieve total opioid-free general anesthesia without locoregional blocks. Best Pract Res Clin Anaesthesiol 31(4): 533-545.

10. Tong CMC, Lucas J, Shah A, Foote C, Simhan J (2018) Novel Multi-Modal Analgesia Protocol Significantly Decreases Opioid Requirements in Inflatable Penile Prosthesis Patients. J Sex Med 15(8): 1187-1194.

11. Brandal D, Keller MS, Lee C, Grogan T, Fujimoto Y, et al. (2017) Impact of Enhanced Recovery After Surgery and Opioid-FreeAnesthesia on Opioid Prescriptions at Discharge from the Hospital: A Historical-Prospective Study. Anesth Analg 125(5): 1784-1792.

12. Lavand'homme P, Estebe JP (2017) Opioid-free anesthesia opioid side effects: Tolerance and hyperalgesia. Best Pract Res Clin Anaesthesiol 31(4): 487-498.

13. Hontoir S, Saxena S, Gatto P, Khalife M, Ben Aziz AM, et al. (2016) Opioid-free anesthesia: what about patient comfort? A prospective, randomized, controlled trial. Acta Anaesthesiol Belg 67(4): 183-190.

14. Kirschen GW, Kim E, Adsumelli RSN (2019) Dexmedetomidine-Induced Massive Diuresis in a Patient Undergoing Spinal Fusion Surgery: A Case Report and Synthesis of the Literature. A A Pract 12(4): 112114.

15. Song Y, Shim JK, Song JW, Kim EK, Kwak YL (2016) Dexmedetomidine added to an opioid-based analgesic regimen for the prevention of postoperative nausea and vomiting in highly susceptible patients: A randomised controlled trial. Eur J Anaesthesiol 33(2): 75-83.

16. Gong Z, Li J, Zhong Y, Guan X, Huang A, et al. (2018) Effects of dexmedetomidine on postoperative cognitive function in patients undergoing coronary artery bypass grafting. Exp Ther Med 16(6): 4685-4689.

17. Bakan M, Umutoglu T, Topuz U, Uysal H, Bayram M, et al. (2015) Opioid-free total intravenous anesthesia with propofol, dexmedetomidine and lidocaine infusions for laparoscopic cholecystectomy: a prospective, randomized, double-blinded study. Braz J Anesthesiol 65(3): 191199.

18. Ghai B, Grandhe RP, Kumar A, Chari P (2005) Comparative evaluation of midazolam and ketamine with midazolam alone as oral premedication. Paediatr Anaesth 15(7): 554-559.

19. Williamson A, Hoggart B (2005) Pain: a review of three commonly used pain rating scales. J Clin Nurs 14(7): 798-804.

20. Leas DP, Connor PM, Schiffern SC, D'Alessandro DF, Roberts KM, et al. (2019) Opioid-free shoulder arthroplasty: a prospective study of a novel clinical care pathway. J Shoulder Elbow Surg S10582746(19)30062-X.

21. Soffin EM, Wetmore DS, Beckman JD, Sheha ED, Vaishnav AS, et al. (2019) Opioid-free anesthesia within an enhanced recovery after surgery pathway for minimally invasive lumbar spine surgery: a retrospective matched cohort study. Neurosurg Focus 46(4): E8.

22. Mulier JP (2019) Is opioid-free general anesthesia for breast and gynecological surgery a viable option? Curr Opin Anaesthesiol 32(3): 257-262.

23. Mulier JP, Dillemans B (2019) Anaesthetic Factors Affecting Outcome After Bariatric Surgery, a Retrospective Levelled Regression Analysis. Obes Surg 29(6): 1841-1850.

24. Grape S, Kirkham KR, Frauenknecht J, Albrecht E (2019) Intra-operative analgesia with remifentanil vs. dexmedetomidine: a systematic review and meta-analysis with trial sequential analysis. Anaesthesia 74(6): 793-800. 
25. Díaz-Crespo J, Malo-Manso A, Bustamante-Domínguez C, Escalona-Belmonte JJ, Cruz-Mañas J, et al. (2018) Laparotomy in a patient under opioid free anesthesia. A Sist Sanit Navar 41(2): 259-262.

26. Frauenknecht J, Kirkham KR, Jacot-Guillarmod A, Albrecht E (2019) Analgesic impact of intra-operative opioids vs. opioid-free anaesthesia: a systematic review and meta-analysis. Anaesthesia 74(5): 651662.

27. Guo YJ, Shi XD, Fu D, Yang Y, Wang YP, et al. (2013) Analgesic effects of the COX-2 inhibitor parecoxib on surgical pain through suppression of spinal ERK signaling. Exp Ther Med 6(1): 275-279.

28. Takaku M, da Silva AC, Iritsu NI, Vianna PTG, Castiglia YMM (2018) Effects of a Single Dose of Parecoxib on Inflammatory Response and Ischemic Tubular Injury Caused by Hemorrhagic Shock in Rats. Pain Res Treat: 8375746.

29. Funai Y, Pickering AE, Uta D, Nishikawa K, Mori T, et al. (2014) Systemic dexmedetomidine augments inhibitory synaptic transmission in the superficial dorsal horn through activation of descending noradrenergic control: an in vivo patch-clamp analysis of analgesic mechanisms Pain 155(3): 617-628.

30. Yamakita S, Matsuda M, Yamaguchi Y, Sawa T, Amaya F (2017) Dexmedetomidine prolongs levobupivacaine analgesia via inhibition of inflammation and p38 MAPK phosphorylation in rat dorsal root ganglion. Neuroscience 361: 58-68.
31. Jebaraj B, Ramachandran R, Rewari V, Trikha A, Chandralekha, et al. (2017) Feasibility of dexmedetomidine as sole analgesic agent during robotic urological surgery: A pilot study. J Anaesthesiol Clin Pharmacol 33(2): 187-192.

32. Zhang YS, Jin LJ, Zhou X, Liu Y, Li Y, et al. (2018) Effect of dexmedetomidine on stress reactions and cellular immune function of patients in perioperative period following radial resection for rectal carcinoma. Biol Regul Homeost Agents 32(1): 139-145.

33. Cui W, Wang S, Han R, Wang Q, Li J (2016) CaMKII Phosphorylation in Primary Somatosensory Cortical Neurons is Involved in the Inhibition of Remifentanil-induced Hyperalgesia by Lidocaine in Male Sprague-Dawley Rats. J Neurosurg Anesthesiol 28(1): 44-50.

34. Kurabe M, Furue H, Kohno T (2016) Intravenous administration of lidocaine directly acts on spinal dorsal horn and produces analgesic effect: An in vivo patch-clamp analysis. Sci Rep 6: 26253.

35. Nakhli MS, Kahloul M, Guizani T, Zedini C, Chaouch A, et al. (2018) Intravenous lidocaine as adjuvant to general anesthesia in renal surgery. Libyan J Med 13(1): 1433418.

36. Soto G, Naranjo González M, Calero F (2018) Intravenous lidocaine infusion. Rev Esp Anestesiol Reanim 65(5): 269-274.

\section{Your next submission with Juniper Publishers} will reach you the below assets

- Quality Editorial service

- Swift Peer Review

- Reprints availability

- E-prints Service

- Manuscript Podcast for convenient understanding

- Global attainment for your research

- Manuscript accessibility in different formats

( Pdf, E-pub, Full Text, Audio)

- Unceasing customer service

Track the below URL for one-step submission https://juniperpublishers.com/online-submission.php 